\title{
Implementation for coping with sample and instrument effects for reverse Monte Carlo modelling of total scattering data
}

\author{
Y. P. Zhang ${ }^{1}$, I. Levin ${ }^{2}$, V. Krayzman², M. Eremenko² and W. Liü and M. G. Tucker ${ }^{1}$ \\ ${ }^{1}$ Neutron Scattering Division, Oak Ridge National Laboratory, 1 Bethel Valley Road, Oak Ridge, TN 37830, USA, \\ ${ }^{2}$ Materials Measurement Science Division, National Institute of Standards and Technology (NIST), 100 Bureau Drive, Gaithersburg, \\ MD 20899, USA, \\ ${ }^{3}$ Dalian Institute of Chemical Physics, Chinese Academy of Sciences, Shahekou, Dalian 116023, People's Republic of China \\ zhangy3@ornl.gov
}

Reverse Monte Carlo (RMC) model is a powerful tool based on supercell approach, targeting at the structure model that explains comprehensive experimental datasets. Typically, the RMCProfile package can incorporate neutron/X-ray total scattering, Bragg and extended X-ray absorption fine structure (EXAFS) data. For practical implementation, apart from theoretical pattern calculation and structure model adjustment based on metropolis algorithm, there are various effects under certain circumstances that one needs to take into account to avoid artificial effects. Here we are going to introduce several different types of correction that we recently developed and implemented, in the framework of RMCProfile, namely, 1) the correction for nano-size effect concerning total scattering modelling for nano-systems from 0D nanoparticles to 2D nanosheets [1]. 2) the implementation of arbitrary Bragg peak profile in a tabulated manner, through interacting with Topas software [2]. 3) the correction for finite instrument resolution effect going beyond the conventionally used analytical approach based on Gaussian assumption for peak shape [2]. Through such development and implementation, we hope to extend the scope of application of RMCProfile package for solving structural problems from local perspective. Typically, the implementation of resolution correction enables the modelling to an otherwise-unreachable super-large length scale, e.g., $100 \AA$, following the supercell approach.

(a)

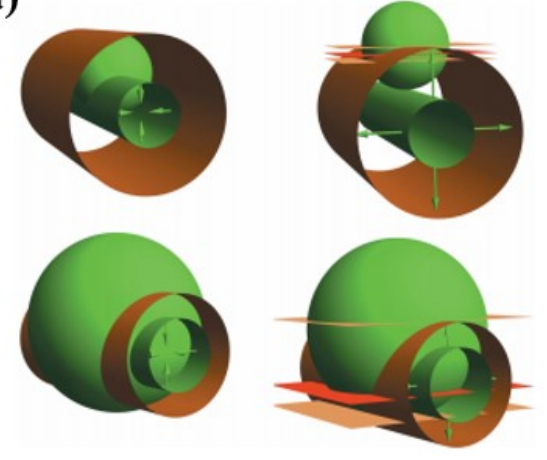

(b)

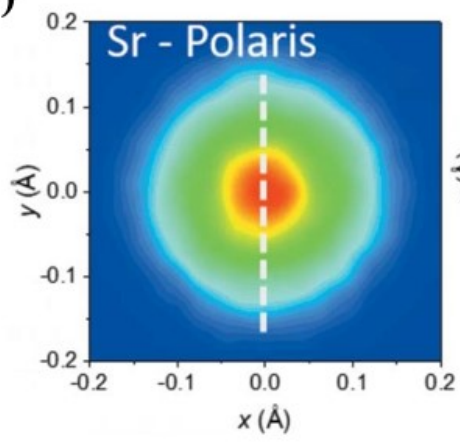

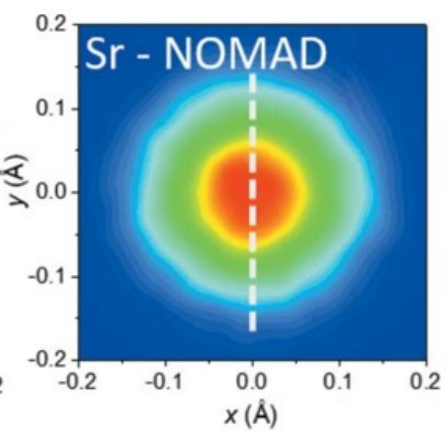

Figure 1. (a) Demo for nano-size effect correction concerning total scattering pattern calculation for 1D nanorod. (b) Comparison of atomic displacement distribution for structure configuration obtained through fitting against data measured at different diffractometers, showing the robustness of our resolution correction routine.

[1] Y. Zhang, M. McDonnell, W. Liu and M. G. Tucker. J. Appl. Cryst. (2019). 52, 1035-1042.

[2] Y. Zhang, M. Eremenko, V. Krayzman, M. G. Tucker and I. Levin. J. Appl. Cryst. (2020). 53, 1509-1518.

Keywords: reverse Monte Carlo; nano-size effect; peak profile tabulation; finite resolution effect. 